\title{
On Green's equivalences in $\Gamma$-groupoids
}

\author{
Kostaq Hila and Jani Dine
}




\title{
ON GREEN'S EQUIVALENCES IN $\Gamma$-GROUPOIDS
}

\author{
KOSTAQ HILA AND JANI DINE
}

Received 24 September, 2013

\begin{abstract}
This paper deals with $\Gamma$-groupoids which are generalizations of groupoids and $\Gamma$ semigroups. The main purpose of this paper is to extend Green's equivalences and Green's Lemma to suitably restricted $\Gamma$-groupoids. We study only $\Gamma$-groupoids satisfying some additional conditions and we show that these are sufficient for the statement of Green's equivalences in case of $\Gamma$-groupoids. Additional condition sufficient to prove Green's Lemma for $\Gamma$-groupoids is provided and some illustrative examples are presented.
\end{abstract}

2010 Mathematics Subject Classification: 20M10; 20M17

Keywords: $\Gamma$-groupoids, $\Gamma$-semigroup, Green's relations

\section{INTRODUCTION AND PRELIMINARIES}

In 1964, N. Nobusawa [16] introduced the notion of a $\Gamma$-ring, more general than a ring. In 1966, W. E. Barnes [1] weakened slightly the conditions in the definition of $\Gamma$-ring in the sense of Nobusawa. Many fundamental results in ring theory have been extended to $\Gamma$-rings by different authors obtaining various generalization analogous to corresponding parts in ring theory. In 1981, M. K. Sen [19] and later in 1986, Sen and Saha [20] introduced the concept of the $\Gamma$-semigroup as a generalization of semigroup and ternary semigroup. Many classical notions and results of the theory of semigroups have been extended and generalized to $\Gamma$-semigroups by a lot of mathematicians. Green's relations for semigroups were introduced by J. A. Green in a paper of 1951 [7]. Green's relations for $\Gamma$-semigroups defined in [6,18], play an important role in studying of the structure of $\Gamma$-semigroups as well as in case of the plain semigroups and become a familiar tool among $\Gamma$-semigroups. Several treatments and contributions concerning Green's relations for $\Gamma$-semigroups have been made by a lot of mathematicians, for instant $[2-4,6,8-10,12,13,15,17,18,20]$. Recently, in [11] we have introduced and studied the hyperversion of Green's relations in $\Gamma$-semihypergroups. The Green's equivalence relations, Green's Lemma and its corollaries are important tools in the theory of $\Gamma$-semigroups as well as in the case of the plain semigroups. The proof of those fundamental results depends on little more than the associativity of the $\Gamma$-operation defined in $\Gamma$-semigroups. However, when we remove this property, we find ourselves faced with the problem of obtaining 
similar results in $\Gamma$-groupoids. For this, we will study only $\Gamma$-groupoids satisfying some additional conditions and we show that these are sufficient for the statement of Green's equivalences in case of $\Gamma$-groupoids. Additional condition sufficient to prove Green's Lemma for $\Gamma$-groupoids is provided and some illustrative examples are presented. The main purpose of this paper is to extend Green's equivalences and Green's Lemma to suitably restricted $\Gamma$-groupoids and to obtain some results which are parallel to those obtained for groupoids and semigroups [5,14].

We introduce below necessary notions and present a few auxiliary results that will be used throughout the paper.

Definition 1. Let $M$ and $\Gamma$ be two non-empty sets. Any map from $M \times \Gamma \times$ $M \rightarrow M$ will be called a $\Gamma$-multiplication in $M$ and denoted by $(\cdot)_{\Gamma}$. The result of this multiplication for $a, b \in M$ and $\alpha \in \Gamma$ is denoted by $a \alpha b$. A $\Gamma$-groupoid $M$ is an ordered pair $\left(M,(\cdot)_{\Gamma}\right)$ where $M$ and $\Gamma$ are non-empty sets and $(\cdot)_{\Gamma}$ is a $\Gamma$-multiplication on $M$.

$M$ is called a $\Gamma$-semigroup, if in addition, the following assertion is satisfied:

$$
\forall(a, b, c, \alpha, \beta) \in M^{3} \times \Gamma^{2},(a \alpha b) \beta c=a \alpha(b \beta c) .
$$

Example 1. Let $\mathrm{M}$ be a semigroup and $\Gamma$ be any nonempty set. If we define $a \gamma b=a b$ for all $a, b \in M$ and $\gamma \in \Gamma$. Then $M$ is a $\Gamma$-semigroup.

Example 2. Let $M$ be a set of all negative rational numbers. Obviously $M$ is not a semigroup under usual product of rational numbers. Let $\Gamma=\left\{-\frac{1}{p}: p\right.$ is prime $\}$. Let $a, b, c \in M$ and $\alpha \in \Gamma$. Now if $a \alpha b$ is equal to the usual product of rational numbers $a, \alpha, b$, then $a \alpha b \in M$ and $(a \alpha b) \beta c=a \alpha(b \beta c)$. Hence $M$ is a $\Gamma$-semigroup.

Example 3. Let $M=\{-i, 0, i\}$ and $\Gamma=M$. Then $M$ is a $\Gamma$-semigroup under the multiplication over complex numbers while $M$ is not a semigroup under complex number multiplication.

Notice that every semigroup is a $\Gamma$-semigroup and $\Gamma$-semigroups are a generalization of semigroups. The same holds for $\Gamma$-groupoids.

A $\Gamma$-groupoid $M$ is said to be commutative if for all $a, b \in M, \gamma \in \Gamma, a \gamma b=b \gamma a$.

\section{ON GREEN'S RELATIONS IN $\Gamma$-GROUPOIDS}

Let $M$ be a $\Gamma$-groupoid. If $\varepsilon$ is any binary relation on the set $M$ and $a, b \in$ $M$, then let $a \mathscr{E} b$ mean that $a$ is $\mathcal{E}$-related to $b$ and, whenever $\mathscr{E}$ is an equivalence relation on $M$, let the $\mathscr{E}$-equivalence class containing $a$ be denoted by $E_{a}$, i.e., $E_{a}=$ $\{x \in M \mid x \& a\}$. We define now two relations, the so-called Green's relations on a $\Gamma$-groupoid $M$. 
Definition 2. Let $M$ be a $\Gamma$-groupoid and $a, b \in M$. We define $a \mathcal{R} b$ if and only if either $a=b$ or there exist $x, y \in M$ and $\alpha, \beta \in \Gamma$, such that $a \alpha x=b$ and $b \beta y=a$. Dually, we define $c \mathscr{L} d$ if and only if either $c=d$ or there exist $u, v \in M$ and $\gamma, \delta \in \Gamma$ such that $u \gamma c=d$ and $v \delta d=c$.

When these are equivalence relations we will write $R_{a}$ for the $\mathcal{R}$-class of $a$, and $L_{c}$ for the $\mathscr{L}$-class of $c$.

When $M$ is associative, that is, a $\Gamma$-semigroup, then it is known that the relations $\mathcal{R}$ and $\mathscr{L}$ are equivalence relations [6]. In this case we have $a \mathcal{R} b$ iff $a \Gamma M \cup\{a\}=$ $b \Gamma M \cup\{b\}$.

For arbitrary $\Gamma$-groupoids, these two subsets of $M$ need not have any particular relationship even though $a \mathcal{R} b$. For this, we give the following definition.

Definition 3. Let $M$ be a $\Gamma$-groupoid. $\mathrm{M}$ is said to be left consistent if $H \gamma(x \alpha y)=$ $(H \gamma x) \alpha y$ for any $x, y \in M, \gamma, \alpha \in \Gamma$ and any $\Gamma$-subgroupoid $H$ of $M . M$ is said to be weakly left consistent if the above holds just for $H=M$.

Definition 4. Let $M$ be a $\Gamma$-groupoid. $\mathrm{M}$ is said to be right consistent if ( $x \alpha y) \gamma H$ $=x \alpha(y \gamma H)$ for any $x, y \in M, \alpha, \gamma \in \Gamma$ and any $\Gamma$-subgroupoid $H$ of $M . M$ is said to be weakly right consistent if the above holds just for $H=M$.

Definition 5. Let $M$ be a $\Gamma$-groupoid. $\mathrm{M}$ is said to be [weakly]consistent if it is both [weakly] left and [weakly] right consistent.

Definition 6. Let $M$ be a $\Gamma$-groupoid. $\mathrm{M}$ is said to be intra-consistent if $(x \gamma H) \alpha y$ $=x \gamma(H \alpha y)$ for any $x, y \in M, \gamma, \alpha \in \Gamma$ and any $\Gamma$-subgroupoid $H$ of $M . M$ is said to be weakly intra-consistent if the above holds just for $H=M$.

Proposition 1. Let $M$ be a weakly right consistent or a weakly intra-consistent $\Gamma$-groupoid. Then $a \mathcal{R} b$ if and only if $a \Gamma M \cup\{a\}=b \Gamma M \cup\{b\}$ for $a, b \in M$.

Proof. " $\Rightarrow "$. Let $M$ be weakly right consistent and assume $a \mathcal{R} b$. If $a=b$ the result is evident. Otherwise there exist $x, y \in M$ and $\alpha, \beta \in \Gamma$ such that $a \alpha x=b$ and $b \beta y=a$. Let $\gamma \in \Gamma$ and so $a \gamma M \subseteq a \Gamma M$. Then we have: $a \gamma M=(b \beta y) \gamma M=$ $b \beta(y \gamma M) \subseteq b \beta M \subseteq b \Gamma M$, that is, $a \Gamma M \subseteq b \Gamma M$. On the other side, we have: $b \gamma M=(a \alpha x) \gamma M=a \alpha(x \gamma M) \subseteq a \alpha M \subseteq a \Gamma M$, that is $b \Gamma M \subseteq a \Gamma M$. Hence $a \Gamma M=b \Gamma M$. Since $a \in b \Gamma M$ and $b \in a \Gamma M$, the requested result follows. If $M$ is weakly intra-consistent and $a \mathcal{R} b$ and $a \neq b$ we can show in a similar way that $a \in a \Gamma M=b \Gamma M$, and $b \in b \Gamma M$, and the result follows immediately.

$" \Leftarrow "$. The converse is trivial.

An immediate corollary of the above proposition is the following.

Corollary 1. If $M$ is either a weakly consistent or a weakly intra-consistent $\Gamma$ groupoids, $\mathcal{R}$ and $\mathscr{L}$ are equivalence relations. Indeed if $M$ is weakly consistent, then $\mathcal{R}$ is a left congruence and $\mathscr{L}$ is a right congruence. 
Problem 1. In general, $\mathcal{R}$ need not be a left congruence on a weakly intraconsistent $\Gamma$-groupoid. Can one finds an example of a non-trivial weakly intraconsistent $\Gamma$-groupoid in which $\mathcal{R}$ is not a left congruence?

Let we consider now the case $M$ is a commutative $\Gamma$-groupoid.

Proposition 2. Let $M$ be a commutative $\Gamma$-groupoid. Then $M$ is [weakly] left consistent if and only if $M$ is [weakly] right consistent and therefore [weakly] consistent.

Proof. Let $a, b \in M, \alpha, \beta \in \Gamma$ and $H$ be any $\Gamma$-subgroupoid of $M$. By the commutativity of $M$ we have $(a \alpha b) \beta H=H \beta(b \alpha a)$ and $a \alpha(b \beta H)=(b \beta H) \alpha a=$ $=(H \beta b) \alpha a$. The two equalities are linked if $M$ is either [weakly] left or [weakly] right consistent and hence the conditions [with $H=M$ ] are equivalent.

Remark 1. For the commutative groupoids, if they are [weakly] right consistent and therefore [weakly] consistent, in either case they are [weakly] intra-consistent. When we pass to $\Gamma$-groupoids, this property does not hold. In fact, if $M$ is [weakly] right (or left) consistent $\Gamma$-groupoid, by the commutativity of $M$, we have: $(a \alpha H) \beta b=$ $b \beta(a \alpha H)=(b \beta a) \alpha H=(a \beta b) \alpha H=a \beta(b \alpha H)=a \beta(H \alpha b)$, for any $a, b \in M, \alpha, \beta \Gamma$ and $H$ is any $\Gamma$-subgroupoid of $M$ [with $H=M$ ], which shows that $M$ is not [weakly] intra-consistent in general.

Example 4. Let $M=\{x, y, z, t\}$ and $\Gamma=\{\alpha, \beta\}$ with the $\Gamma$-multiplication defined by

\begin{tabular}{l|lllll|llll}
$\alpha$ & $x$ & $y$ & $z$ & $t$ & $\alpha$ & $x$ & $y$ & $z$ & $t$ \\
\hline$x$ & $x$ & $x$ & $y$ & $y$ & $x$ & $x$ & $x$ & $y$ & $y$ \\
$y$ & $y$ & $y$ & $x$ & $x$ & $y$ & $y$ & $y$ & $x$ & $x$ \\
$z$ & $z$ & $z$ & $t$ & $t$ & $z$ & $t$ & $t$ & $z$ & $z$ \\
$t$ & $t$ & $t$ & $z$ & $z$ & $t$ & $z$ & $z$ & $t$ & $t$
\end{tabular}

It can be easily verified that $M$ is weakly left consistent and weakly intra-consistent but not weakly right consistent.

Problem 2. Can one find an example of a non-trivial $\Gamma$-groupoid which is both weakly left and right consistent but not weakly intra-consistent or to prove that a weakly consistent $\Gamma$-groupoid is weakly intra-consistent?

In order to prove Green's Lemma for $\Gamma$-groupoids we will need the following result, whose proof is straightforward.

Lemma 1. Let $M$ be a $\Gamma$-groupoid. If $M$ is weakly right consistent, then for all $a \in M$ and $\rho \in \Gamma$, a $\rho$ is a $\Gamma$-subgroupoid. Also, $a \Gamma M$ is a $\Gamma$-subgroupoid.

Corollary 2. Let $M$ be a $\Gamma$-groupoid. If $M$ is weakly right consistent, then for all $a \in M, \rho \in \Gamma, a \rho M \cup\{a\}$ is a $\Gamma$-subgroupoid. Also, $a \Gamma M \cup\{a\}$ is a $\Gamma$-subgroupoid. 
Let $M$ be a $\Gamma$-groupoid. The mapping $\lambda_{\alpha}^{s}: M \rightarrow M$ defined by $\lambda_{\alpha}^{s} a=s \alpha a$ for all $s, a \in M$ and $\alpha \in \Gamma$ is called left translation of $\Gamma$-semigroup $M$. The mapping $\rho_{s^{\prime}}^{\beta}: M \rightarrow M$ defined by $b \rho_{s^{\prime}}^{\beta}=b \beta s^{\prime}$ for all $s^{\prime}, b \in M$ and $\beta \in \Gamma$ is called right translation of $\Gamma$-semigroup $M$.

Theorem 1. Let $M$ be a consistent $\Gamma$-groupoid and suppose $c \mathcal{R} b$ for some $c \neq$ $b$. Then there are $s, s^{\prime} \in M, \alpha, \beta \in \Gamma$ such that $c \alpha s=b, b \beta s^{\prime}=c$ and the right translations $\rho_{s}^{\alpha}, \rho_{s^{\prime}}^{\beta}$, are, respectively, mappings from $L_{c}$ into $L_{b}$ and $L_{b}$ into $L_{c}$, which are $\mathcal{R}$-class preserving, that is, for $x \in L_{c}, x \mathcal{R} x \rho_{s}^{\alpha}$ and for $y \in L_{b}, y \mathcal{R} y \rho_{s^{\prime}}^{\beta}$.

Proof. Let $c \mathcal{R} b$ for some $c \neq b$. Since $c \neq b$, the existence of $s, s^{\prime}$ follows from the Definition 2. Now let $a \mathscr{L} c$ and $d \mathscr{L} b$. By Corollary $1, \mathscr{L}$ is a right congruence, thus we have a a s $\mathscr{L} c \alpha s=b$ and $d \beta s^{\prime} \mathscr{L} b \beta s^{\prime}=c$. Thus we have $L_{c} \rho_{s}^{\alpha} \subseteq L_{b}$ and $L_{b} \rho_{s^{\prime}}^{\beta} \subseteq L_{c}$.

Now, if $a \neq c$, then for any $\rho \in \Gamma, a \Gamma M \supseteq a \alpha(s \rho M)=(t \gamma c) \alpha(s \rho M)$ where $t \gamma c=a$ for some $t \in M, \gamma \in \Gamma$ by the Definition 2. Since $s \rho M$ is a $\Gamma$-subgroupoid by Lemma 1, we have $(t \gamma c) \alpha(s \rho M)=t \gamma[c \alpha(s \rho M)]=t \gamma[(c \alpha s) \rho M]=t \gamma(b \rho M)$. Thus $a=t \gamma c=t \gamma\left(b \beta s^{\prime}\right) \in t \gamma(b \Gamma M) \subseteq a \Gamma M$. Continuing we have $a \Gamma M \supseteq(a \alpha s) \rho M=t \gamma(b \rho M) \supseteq t \gamma\left[b \beta\left(s^{\prime} \rho M\right)\right]=t \gamma\left[\left(b \beta s^{\prime}\right) \rho M\right]=t \gamma(c \rho M)=$ $(t \gamma c) \rho M=a \rho M$, whence $a \Gamma M=(a \alpha s) \Gamma M$. But $a \in a \Gamma M$ and $a \alpha s \in a \Gamma M=$ $(a \alpha s) \Gamma M$ so that we can conclude by Proposition 1 that $a \mathcal{R} a \alpha s$. If $a=c$, the preceding argument can be simplified to show that $c \mathcal{R} c \alpha s$. In a similar way, it can be shown $d \mathcal{R} d \beta s^{\prime}$.

Corollary 3. If $M$ is a consistent $\Gamma$-groupoid, then $\mathcal{R} \circ \mathscr{L}=\mathscr{L} \circ \mathcal{R}$ on $M$.

Proof. If $a \mathscr{L} c \mathcal{R} b$, then the above Theorem yields an $s \in M$ such that $a \mathcal{R} a \gamma s \mathscr{L} b$ for some $\gamma \in \Gamma$, and so $\mathscr{L} \circ \mathcal{R} \subseteq \mathcal{R} \circ \mathscr{L}$. The reverse inclusion is proven dually.

Now we give the following definition.

Definition 7. A $\Gamma$-groupoid $M$ is said to be $\mathscr{D}-\Gamma$-groupoid if $\mathscr{L} \circ \mathcal{R}=\mathcal{R} \circ \mathscr{L}$ on $M$.

In such cases we define $\mathscr{D}=\mathscr{L} \circ \mathcal{R}$ and $\mathscr{D}$ is then clearly an equivalence relation.

Note that the consistent $\Gamma$-groupoids are $\mathscr{D}-\Gamma$-groupoid, while the converse is not necessarily true. The following example shows this.

Example 5. Let $M=\{x, y\}$ and $\Gamma=\{\alpha, \beta\}$ with the $\Gamma$-multiplication defined by

\begin{tabular}{|c|c|c|c|c|c|}
\hline$\alpha$ & $x$ & $y$ & $\beta$ & $x$ & $y$ \\
\hline$x$ & $y$ & $x$ & $x$ & $x$ & $x$ \\
\hline$y$ & $y$ & $x$ & $y$ & $x$ & $x$ \\
\hline
\end{tabular}

Here we have that $\mathscr{L}=\mathcal{R}=\omega$, the universal relation on $M$, so that $M$ is certainly $D-\Gamma$-groupoid, while $M \alpha(x \beta x)=y \neq x=(M \alpha y) \beta y$, that is, $M$ is not a consistent $\Gamma$-groupoid. 


\section{GREEN'S LEMMA FOR $\Gamma$-GROUPOIDS}

Theorem 1 shows us that for a consistent $\Gamma$-groupoid $M$ certain right translations define maps between two $\mathscr{L}$-classes which are $\mathcal{R}$-class preserving. In general, it is not known if $\rho_{s}^{\alpha}$ and $\rho_{s^{\prime}}^{\beta}$ are mutually inverse maps between $L_{c}$ and $L_{b}$, as is the case of $\Gamma$-semigroups [6].

In the following results are provided additional conditions which suffice to guarantee this result.

Definition 8. Let $M$ be a $\mathscr{D}-\Gamma$-groupoid. A $\mathscr{D}$-class, $D$ of $M$ is said to be regular if there is an $\gamma$-idempotent element $(x \gamma x=x)$ in each $\mathscr{L}$ and $\mathcal{R}$-class of $D$.

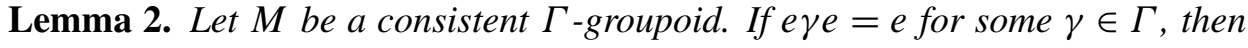
$x \gamma e=x$ for all $x \in \mathscr{L}$-class, $L_{e}$, and $e \gamma y=y$ for all $y \in \mathcal{R}$-class $R_{e}$.

Proof. Let $x \in L_{e}$. Then $x=t \alpha e$ for some $t \in M$ and $\alpha \in \Gamma$. Now $x \gamma e=$ $(t \alpha e) \gamma e=t \alpha(e \gamma e)=t \alpha e=x$ since $\{e\}$ is a $\Gamma$-subgroupoid of $M$. The other result is dual.

Proposition 3. Let $D$ be a regular $D$-class of a consistent $\Gamma$-groupoid $M$. Then for any $a \in D$, there exist $t, t^{\prime} \in M$ and $\gamma, \gamma^{\prime}, \alpha, \beta \in \Gamma$ such that $a=a \gamma(t \alpha a)=$ $\left(a \beta t^{\prime}\right) \gamma^{\prime} a$.

Proof. Let $a \in D$. Since $D$ is regular, there is an $\gamma$-idempotent $e \in L_{a}=L_{e}$. By

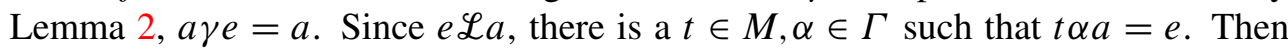
$a=a \gamma e=a \gamma(t \alpha a)$. Dually one obtains $a=\left(a \beta t^{\prime}\right) \gamma^{\prime} a$.

Remark 2. A converse of Proposition 3 is false: a $\Gamma$-groupoid $M$ may be consistent, and for every $a \in M$ may have $t, t^{\prime} \in M$ and $\gamma, \gamma^{\prime}, \alpha, \beta \in \Gamma$ such that $a=$ $a \gamma(t \alpha a)=\left(a \beta t^{\prime}\right) \gamma^{\prime} a$, and yet $M$ may have no idempotents. We have the following example.

Example 6. Let $M=\{x, y\}$ and $\Gamma=\{\alpha, \beta\}$ with the $\Gamma$-multiplication defined by

\begin{tabular}{|c|c|c|c|c|c|}
\hline$\alpha$ & $x$ & $y$ & $\beta$ & $x$ & $y$ \\
\hline$x$ & $y$ & $x$ & $x$ & $y$ & $y$ \\
\hline$y$ & $y$ & $x$ & $y$ & $x$ & $x$ \\
\hline
\end{tabular}

In the $\Gamma$-groupoid $M$ there no idempotents and further, for example, $x=x \alpha(x \beta x)=$ $(x \alpha x) \beta x$. Moreover, here we have $\mathscr{L}=\mathcal{R}=\omega$.

Definition 9. Let $M$ be a $\Gamma$-groupoid. $M$ is said to be almost associative if whenever $H$ is a $\Gamma$-subgroupoid of $M$ and $a, b, c \in M, \alpha, \beta, \gamma \in \Gamma$, we have $H \gamma[(a \alpha b) \beta c]=H \gamma[a \alpha(b \beta c)]$ and $[a \alpha(b \beta c)] \gamma H=[(a \alpha b) \beta c] \gamma H$.

Theorem 2. A regular, consistent, almost associative $\Gamma$-groupoid is associative. 
Proof. Let $a, b, c \in M$. Then $(a \alpha b) \beta c=(a \alpha b) \beta(c \gamma e)$ for some eje $=e \in$ $L_{c}, \gamma \in \Gamma$. But $(a \alpha b) \beta(c \gamma e)=[(a \alpha b) \beta c] \gamma e=[a \alpha(b \beta c)] \gamma e=a \alpha[(b \beta c) \gamma e]=$ $a \alpha[b \beta(c \gamma e)]=a \alpha(b \beta c)$ since $M$ is consistent and almost associative and $\{e\}$ is a $\Gamma$-subgroupoid. We thus have that $(a \alpha b) \beta c=a \alpha(b \beta c)$ for any $a, b, c \in M$ and $\alpha, \beta \in \Gamma$, i.e., $M$ is associative.

Now, based on the above results, we are ready to state and to prove the so-called Green's Lemma for $\Gamma$-groupoids.

Corollary 4. (Green's Lemma). Let $M$ be a consistent almost associative $\Gamma$ groupoid. If $D$ is a regular $D$-class of $M$ and $c \mathcal{R} b$ for $c, b \in D$, then there exist $s, s^{\prime} \in M, \alpha, \beta \in \Gamma$ such that $c \alpha s=b, b \beta s^{\prime}=c$ and the right translations $\rho_{s}^{\alpha}, \rho_{s^{\prime}}^{\beta}$, are mutually inverse bijections between $L_{c}$ and $L_{b}$ and are $\mathcal{R}$-class preserving.

Proof. By Theorem 1 we need only to show that $\rho_{s}^{\alpha}$ and $\rho_{s^{\prime}}^{\beta}$ are mutually inverse bijections. Let $f \gamma f=f \in R_{c}$ for some $\gamma \in \Gamma$, and $f \gamma u=c$ for some $u \in M$. Then $c=b \beta s^{\prime}=(c \alpha s) \beta s^{\prime}=((f \gamma u) \alpha s) \beta s^{\prime}=(f \gamma(u \alpha s)) \beta s^{\prime}=f \gamma\left((u \alpha s) \beta s^{\prime}\right)=$ $f \gamma\left(u \alpha\left(s \beta s^{\prime}\right)\right)=(f \gamma u) \alpha\left(s \beta s^{\prime}\right)=c \alpha\left(s \beta s^{\prime}\right)$.

Now let $a \in L_{c}$ and $e \delta e=e \in L_{c}$ for some $\delta \in \Gamma$. There exist $t \in M$ and $\mu \in \Gamma$ such that $t \mu c=a$. Then $(a) \rho_{s}^{\alpha} \rho_{s^{\prime}}^{\beta}=(a \alpha s) \beta s^{\prime}$. Since $(a \alpha s) \beta s^{\prime} \in L_{a} \cap$ $R_{a}$ by Theorem 1, $(a \alpha s) \beta s^{\prime}=\left[(a \alpha s) \beta s^{\prime}\right] \delta e=\left[a \alpha\left(s \beta s^{\prime}\right)\right] \delta e=\left[(t \mu c) \alpha\left(s \beta s^{\prime}\right)\right] \delta e=$ $\left[t \mu\left(c \alpha\left(s \beta s^{\prime}\right)\right)\right] \delta e=[t \mu c] \delta e=a \delta e=a$ since $c=c \alpha\left(s \beta s^{\prime}\right)$ from above. Thus $\rho_{s}^{\alpha} \rho_{s^{\prime}}^{\beta}$ is the identity map on $L_{c}$. Similarly $\rho_{s^{\prime}}^{\beta} \rho_{s}^{\alpha}$ is the identity map on $L_{b}$. The result now follows.

It is clear that, as it is shown in this paper, a consistent, almost associative $\Gamma$ groupoid with every $(\mathcal{R} \circ \mathscr{L})$-equivalence class regular is necessarily a $\Gamma$-semigroup and so the Green's Lemma takes its familiar form in $\Gamma$-semigroups [3, 6].

From all the above, the following problems arise:

Problem 3. What we can say about the validation of Green's Lemma for infinite $\Gamma$-groupoids?

Problem 4. How necessary is the regularity in the Corollary 4?

Problem 5. Is there any natural condition for $\Gamma$-groupoids weaker than that of almost associativity which can replace it leaving Corollary 4 true but Theorem 2 false?

Problem 6. Is there a regular consistent $\Gamma$-groupoid in which the right translations of Theorem 1 are not mutually inverse?

\section{ACKNOWLEDGEMENT}

The authors would like to express their warmest thanks to the referee for his time to read the manuscript and his useful suggestions. 


\section{REFERENCES}

[1] W. E. Barnes, "On the $\Gamma$-rings of Nobusawa," Pac. J. Math., vol. 18, pp. 411-422, 1966.

[2] R. Chinram and P. Siammai, "On Green's relations for $\Gamma$-semigroups and reductive $\Gamma$ semigroups," Int. J. Algebra, vol. 2, no. 1-4, pp. 187-195, 2008.

[3] R. Chinram and P. Siammai, “Green's lemma and Green's theorem for $\Gamma$-semigroups," Lobachevskii J. Math., vol. 30, no. 3, pp. 208-213, 2009.

[4] N. Chunse and M. Siripitukdet, "The Natural partial order on regular $\Gamma$-semigroups," Bull. Malaysian Math. Sci. Soc., accepted.

[5] A. H. Clifford and G. B. Preston, The algebraic theory of semigroups. Vol. 1. Providence, R.I., USA: American Mathematical Society, 1961.

[6] T. K. Dutta and T. K. Chatterjee, "Green's equivalences on $\Gamma$-semigroup," Bull. Calcutta Math. Soc., vol. 80, no. 1, pp. 30-35, 1988.

[7] J. A. Green, "On the structure of semigroups," Ann. of Math. (2), vol. 54, pp. 163-172, 1951.

[8] D. Heidari and M. Amooshahi, "Transformation semigroups associated to $\Gamma$-semigroups," Discuss. Math., Gen. Algebra Appl., vol. 33, no. 2, pp. 249-259, 2013.

[9] D. Heidari and B. Davvaz, " $n$-ary $\Gamma$-semigroups, operators and Green's relations," Afr. Mat., vol. 25, no. 3, pp. 841-856, 2014.

[10] K. Hila, "On regular, semiprime and quasi-reflexive $\Gamma$-semigroup and minimal quasi-ideals," $L o b$ achevskii J. Math., vol. 29, no. 3, pp. 141-152, 2008.

[11] K. Hila, B. Davvaz, and J. Dine, "Study on the structure of $\Gamma$-semihypergroups," Commun. Algebra, vol. 40, no. 8, pp. 2932-2948, 2012.

[12] K. Hila and J. Dine, "On Green's relations, $2^{0}$-regularity and quasi-ideals in $\Gamma$-semigroups," Acta Math. Sin., Engl. Ser., vol. 29, no. 3, pp. 609-624, 2013.

[13] K. Hila and J. Dine, "On the structure of Green's relations in $\mathcal{B Q}-\Gamma$-semigroups," An. Ştiinţ. Univ. Al. I. Cuza Iaşi, Ser. Nouă, Mat., vol. 60, no. 1, pp. 201-210, 2014.

[14] K. M. Kapp, “Green's Lemma for groupoids," Rocky Mt. J. Math., vol. 1, pp. 551-559, 1971.

[15] N. Kehayopulu, "Green's relations and the relation $\mathcal{N}$ in $\Gamma$-semigroups," Quasigroups Relat. Syst., vol. 22, pp. 89-96, 2014.

[16] N. Nobusawa, "On a generalization of the ring theory," Osaka J. Math., vol. 1, pp. 81-89, 1964.

[17] P. Petro and T. Xhillari, "Green's theorem and minimal quasi-ideals in $\Gamma$-semigroups," Int. $J$. Algebra, vol. 5, no. 9-12, pp. 461-470, 2011.

[18] N. K. Saha, “On $\Gamma$-semigroup. II,” Bull. Calcutta Math. Soc., vol. 79, no. 6, pp. 331-335, 1987.

[19] M. K. Sen, "On $\Gamma$-semigroup," in Algebra and its applications. (International Symposium on Algebra and Its Applications, 1981, New Delhi, India)., H. Manocha and J. Srivastava, Eds. New York: Marcel Dekker Publication, 1984, pp. 301-308.

[20] M. K. Sen and N. K. Saha, “On $\Gamma$-semigroup. I,” Bull. Calcutta Math. Soc., vol. 78, pp. 180-186, 1986.

\section{Authors' addresses}

Kostaq Hila

Department of Mathematics \& Computer Science, Faculty of Natural Sciences, University of Gjirokastra, Albania

E-mail address: kostaq_hila@yahoo.com

Jani Dine

Department of Mathematics \& Computer Science, Faculty of Natural Sciences, University of Gjirokastra, Albania

E-mail address: jani_dine@yahoo.com 